\title{
Beam loading assisted matching scheme for high quality plasma acceleration in linear regime
}

\author{
S. Romeo $\odot^{*}$ and M. Ferrario \\ Laboratori Nazionali di Frascati, Via Enrico Fermi 40, 00044 Frascati (Rome), Italy \\ A. R. Rossi \\ INFN-Milan, Via Celoria 16, 20133 Milan, Italy
}

(Received 3 June 2019; accepted 1 July 2020; published 14 July 2020)

\begin{abstract}
We propose and we numerically design a working point for beam driven plasma wakefield acceleration that allows us to preserve the witness quality. This working point is a new scheme where a low density driver generates a wakefield in linear regime and the witness transverse evolution is dominated by the beam loading effect. We derived optimal matching conditions to prevent the phase space degradation by means of a transverse envelope equation.
\end{abstract}

DOI: 10.1103/PhysRevAccelBeams.23.071301

\section{INTRODUCTION}

The next generation of high-energy compact particle colliders will require an increase of several orders of magnitude of the accelerating gradients that are currently used and that are very difficult to obtain using metallic rf structures [1]. Plasma wakefield acceleration proved to be a valid candidate for the achievement of such gradients $[2,3]$. Using a plasma structure as a transformer it is possible to transfer the energy of a driving pulse to an accelerated bunch using the plasma wakefields. The driving pulse exciting the wake can be either a laser pulse [4] (laser wakefield acceleration LWFA) or a particle bunch [5] (particle wakefield acceleration PWFA).

There are several procedures for the generation of the trailing bunch, that can be roughly grouped in internal [6] and external injection schemes [5]. The internal injection occurs when the bunch is generated trapping the plasma electrons in the wake during the propagation of the driving pulse. Instead, we refer to external injection when the bunch is generated by means of a previous accelerating stage and injected inside plasma immediately after the driving pulse. The internal injection scheme provides the synchronization of the driving and trailing pulse. The drawback of this scheme is the weak control of the trailing bunch parameters that are required for high quality acceleration. On the other side, the external injection scheme provides a high degree of control on the trailing bunch parameters at the injection, but

*stefano.romeo@lnf.infn.it.

Published by the American Physical Society under the terms of the Creative Commons Attribution 4.0 International license. Further distribution of this work must maintain attribution to the author(s) and the published article's title, journal citation, and DOI. requires a greater technological effort for the synchronization of the driving pulse with trailing bunch. This issue can be mitigated by means of PWFA with external injection. In this scheme, generally, both the driving bunch (driver) and the trailing bunch (witness) can be generated with the same photoinjector [7,8], guaranteeing a natural synchronization of the pulses. The drawback is a difficult control of the transverse beam dynamics, since the transport line cannot be easily optimized for both bunches at the same time. Therefore, in most cases, the driver has a significantly lower quality than the witness. From this follows a lower driver density at the plasma injection.

In this work we propose a novel scheme for PWFA with external injection with the aim of preserving the witness quality. This scheme involves the use of a low density driver that generates a wakefield in linear regime and a high quality witness with a high density that is injected close to the crest of the accelerating wakefield. Beam loading effects are exploited to keep under control energy spread and to provide transverse focusing. In fact, the crest region of the accelerating wakefield provides the highest accelerating gradient but no focusing field to control the witness transverse evolution. In this scenario, the witness confinement is provided by self-focusing effect.

This configuration has been exploited at some level in a different scenario by the authors in the context of a proposed working point for the SPARC_LAB plasma acceleration experiment [9] and for the AWAKE experiment at CERN [10]. The field generated by the driver can be described analytically using the cold plasma fluid model $[3,11,12]$ that will be used to derive the matching conditions for the preservation of beam quality along the accelerating length.

The idea of using the self focusing field to stabilize the witness transverse evolution arises from the evidence of the self focusing effect experienced by a beam injected inside a 


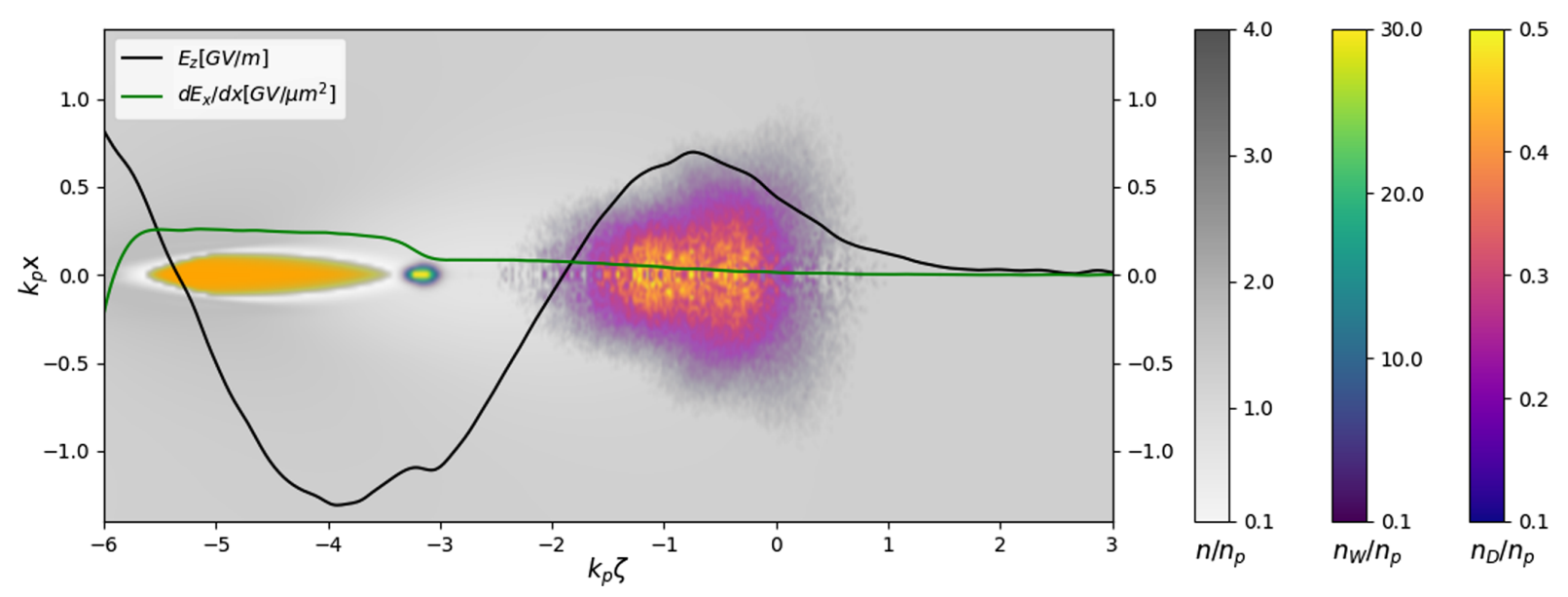

FIG. 1. A simulation of the proposed scheme using the code ARCHITECT. The snapshot is taken at $1 \mathrm{~cm}$ inside the plasma channel. In this case we used a background plasma with a density $n_{p}=2 \times 10^{16} \mathrm{~cm}^{-3}$. The coordinate system is in the reference frame of the laboratory, comoving with the center of the driving bunch. The beams are axially symmetric bi-Gaussian shaped. The driver has a low density $n_{b} / n_{p} \approx 1$, and the witness has a high density $n_{b} / n_{p} \gg 1$. The accelerating field $E_{z}$ is represented in black and the transverse derivative of the focusing field $E_{x}$ is represented in green. The longitudinal compensation of energy spread occurs in correspondence of the witness. The magnitude of the transverse field in this region is lower than in the blowout region (marked in orange behind the witness) and is the result of the combination of the focusing field of the driver and the self-focusing generated by the witness itself.

neutral plasma $[13,14]$. In this configuration, the witness quality is not affected by the driver transverse evolution, preserving a relatively higher stability. The drawback is an accelerating gradient that is slightly lower than the fields generated in blow-out regime at the same plasma density.

Numerical simulations showed that, in a small region close to the witness center, the plasma current is negligible and the local plasma density is approximatively constant, thus the focusing field driving the witness transverse dynamics can be accurately described by the ion column model [14].

We will exploit analytically and by numerical simulations an ideal scenario where the witness quality is preserved by means of the self-focusing effect. We assume a flat top plasma profile with a background density $n_{p}=2 \times 10^{16} \mathrm{~cm}^{-3}$ and ultrarelativistic $(\beta \approx 1)$ driving and trailing electron beams with a bi-Gaussian profile and cylindrical symmetry. In the first part of this paper the transverse matching conditions for a stable driver propagation is determined. In the second part the transverse matching conditions for the witness are evaluated. Scaling laws are discussed and compared for several cases to data from 2D cylindrical simulations with the hybrid fluid kinetic code architect [15].

\section{WORKING POINT DESIGN}

A setup with two beams as shown in Fig. 1 is considered. The beams are chosen with bi-Gaussian spatial distribution of the form $n_{b}(r, \xi)=\alpha n_{p} e^{-r^{2} / \sigma_{r}^{2}} e^{-\xi^{2} / 2 \sigma_{z}^{2}}$ where $\xi=z-c t$, $\alpha=n_{b} / n_{p}$ is the ratio between the beam density and the background plasma density and $\sigma_{r}$ and $\sigma_{z}$ are the transverse and longitudinal rms beam size. The beam parameters are listed in Table I.
We refer to a relatively low energy case $(\gamma=200)$, assuming a driver with low brightness and a witness with high brightness. The corresponding peak densities are $n_{D} \approx$ $n_{p}$ for the driver and $n_{w} \approx 30 n_{p}$ for the witness. The driver is kept at low density in order to generate a wakefield in linear regime. The emittance values are calibrated in order to obtain a stable propagation, and they will be clarified in next sections. The ideal plasma channel that was implemented numerically consists of $5 \mathrm{~cm}$ long flat top that is homogeneous both in longitudinal and transverse direction. The background peak density is $n_{p}=2 \times 10^{16} \mathrm{~cm}^{-3}$. The simulation box is made of $366 \hat{u}_{x} \times 932 \hat{u}_{z}$ grid cells, with $\hat{u}_{z}$ and $\hat{u}_{x}$ versors in the $z$ and $x$ dimension respectively, corresponding to a box dimension of $275 \mu \mathrm{m} \hat{u}_{r} \times 700 \mu \mathrm{m}$ $\hat{u}_{z}$. The driver is placed at $z=0$ and it is discretized with $4 \times 10^{5}$ particles. The witness is placed behind the driver at $z=-117 \mu \mathrm{m}$ and it is discretized with $5 \times 10^{4}$ particles. The integration time step of the simulation is $\Delta t=0.44 \mathrm{fs}$. The mesh is a square which size is $0.75 \mu \mathrm{m} \times 0.75 \mu \mathrm{m}$. These parameters provide an acceptable convergence of the simulation results that does not change significantly varying the mesh size up to $0.25 \mu \mathrm{m} \times 0.25 \mu \mathrm{m}$. In these

TABLE I. Beam parameters at the injection.

\begin{tabular}{lcc}
\hline \hline & Driver & Witness \\
\hline$Q[\mathrm{pC}]$ & 200 & 10 \\
$\gamma$ & 200 & 200 \\
$\epsilon_{n}[\mu \mathrm{m}]$ & 17 & 0.3 \\
$\sigma_{r}[\mu \mathrm{m}]$ & 10.3 & 1.26 \\
$\sigma_{z}[\mu \mathrm{m}]$ & 37.2 & 3 \\
$\sigma_{E}[\%]$ & 0.1 & 0.1 \\
\hline \hline
\end{tabular}



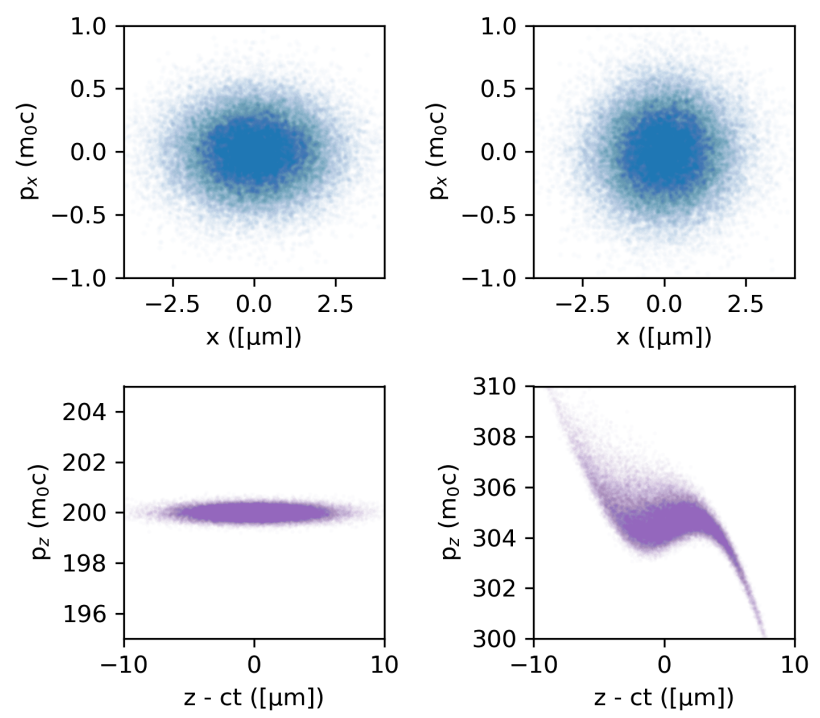

FIG. 2. Witness phase space before acceleration (left) and after acceleration (right). The transverse phase space (blue) does not present any major deformation. The longitudinal phase space (purple) shows that the spread increase occurs mostly in the tails while the moment distribution in the beam core is flat.

trial simulations, the time step accordingly in order to keep a constant CFL (Courant-Friedrichs-Lewy) parameter $[16,17]$ of 0.25 . In Fig. 2 is displayed the longitudinal and transverse phase space of the witness before and after the acceleration. The accelerating gradient is $1.05 \mathrm{GV} / \mathrm{m}$, with an effective energy increase of $\approx 50 \mathrm{MeV}$. The transverse phase space does not show any major alteration. The longitudinal phase space displays a major deformation occurring on the head and on the tail of the beam, while the core is mostly preserved. The evolution of the integrated parameters is shown in Figure 3. What is expected from a nearly matched beam is an oscillation of the $\beta$-function around its equilibrium value $\beta_{m}$ that can be derived as a quasistationary solution of the envelope equation $[18,19]$. Assuming a linear focusing with a normalized focusing strength $K$, the matching $\beta_{m}=K^{-1 / 2}$. In Fig. 3(a) is displayed the $\beta$-function of the witness bunch versus its energy. The betatron oscillations obtained by numerical simulations is compared with the equilibrium $\beta_{m}$ where $K$ is derived by the ion column model [14] decreased of a factor 2, namely

$$
\beta_{m}=\frac{2 \sqrt{\gamma}}{k_{p}}
$$

where $k_{p}=\sqrt{e^{2} n_{p} /\left(\epsilon_{0} m_{e} c^{2}\right)}$ is the plasma wave number. In Fig. 3(b) is displayed the spot size $\sigma_{x}$ and the normalized emittance $\epsilon_{x}$ versus the position of the bunch in the plasma channel. The spot size oscillations are of the order of $0.1 \mu \mathrm{m}$ and the emittance remains constant along the whole accelerating length. For sake of completeness in our analysis the case of a mismatched beam is also included, (a)

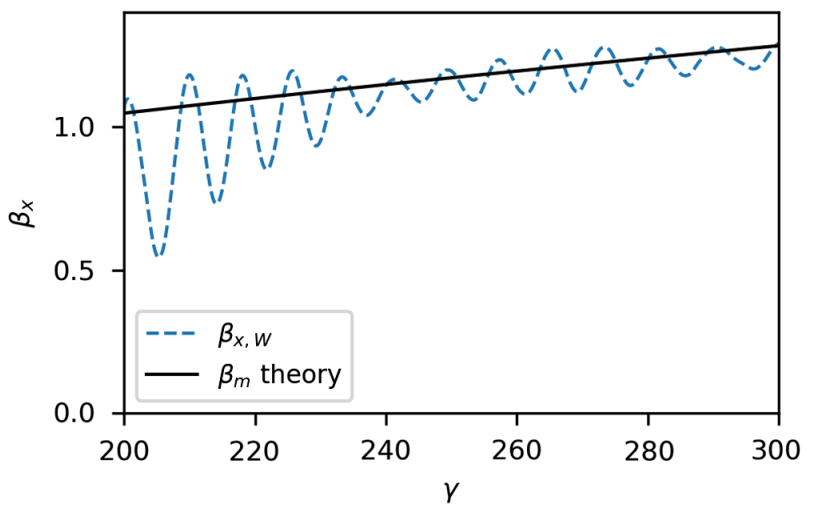

(b)

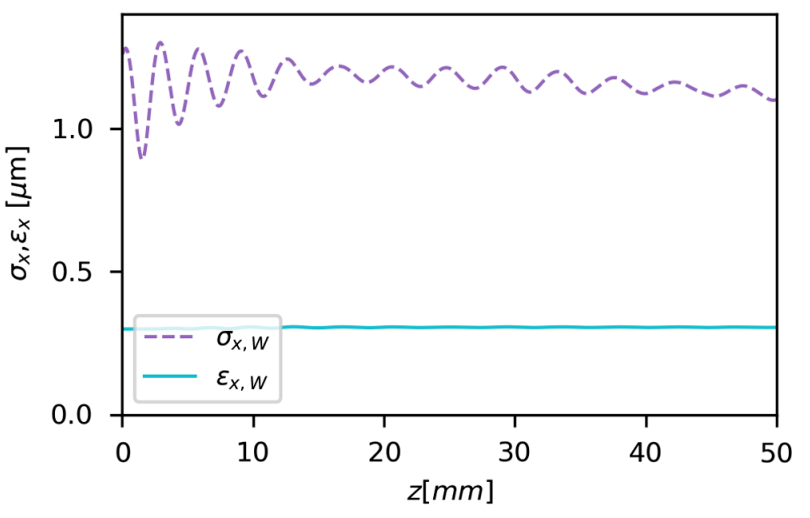

FIG. 3. Transverse evolution of the witness. In (a) is displayed the evolution of the $\beta$ function versus the mean energy $\gamma$ compared to Eq. (1). In (b) is displayed the envelope and normalized emittance evolution.

comparing the simulation results with our main case. The transverse beam dynamics of a mismatched beam is described by betatron dephasing theory [18]. The final emittance of a mismatched case can be written as

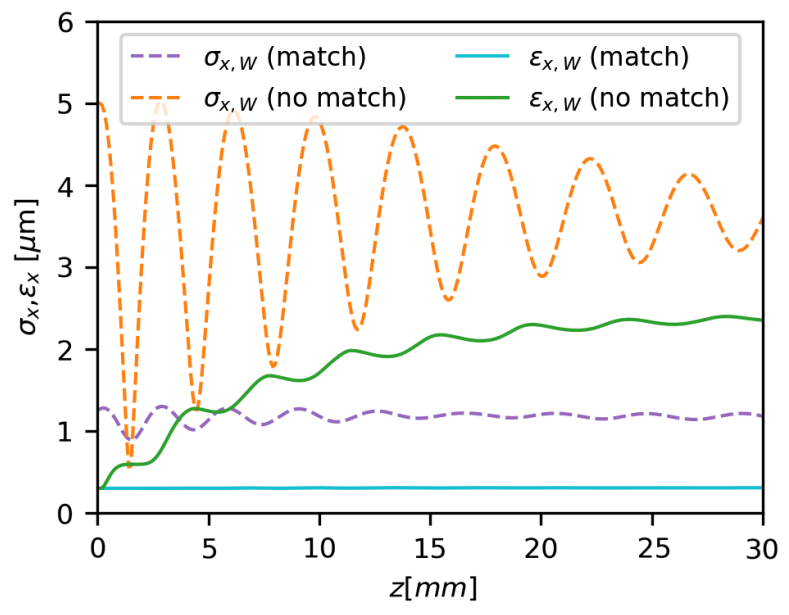

FIG. 4. Comparison between matched (purple) and mismatched (orange) envelope of the witness. The emittance growth of the matched case (cyan) is negligible, while in the mismatched case the emittance (green) grows from the injection value up to a saturation value of $2.4 \mu \mathrm{m}$. 


$$
\epsilon_{n, \mathrm{fin}}=\frac{\epsilon_{n, \text { init }}}{2}\left(\frac{1+\alpha_{\mathrm{inj}}^{2}}{\beta^{*}}+\beta^{*}\right)
$$

where $\alpha_{i n j}$ is the Twiss $\alpha$ function [20] at the injection, $\beta^{*}$ is the normalized $\beta$ defined as the ratio between $\beta_{i n j}$ at the injection and the matched $\beta_{m}, \beta^{*}=\beta_{i n j} / \beta_{m}$. The tested mismatched beam is injected at waist $\alpha_{i n j}=0$ with a transverse spot size $\sigma_{x, y}=5 \mu \mathrm{m}$, corresponding to a $\beta_{i n j}=16.67 \mathrm{~mm}$. The value of $\beta_{m}$ according to Eq. (1) is $\beta_{m}=1.05 \mathrm{~mm}$. According to Eq. (2) this corresponds to an emittance increase from $\epsilon_{n, \text { init }}=0.3 \mu \mathrm{m}$ to the saturation value of $\epsilon_{n \text {,fin }}=2.4 \mu \mathrm{m}$, in total agreement with the results from simulation shown in Fig. 4.

\section{DRIVER MATCHING CONDITIONS}

Driving bunch parameters were chosen in order to maximize the accelerating field and to provide a stable focusing. Since the bunch has a low density, linear theory provides a suitable description of the fields. The maximum accelerating gradient in linear regime PWFA [12] is guaranteed by the resonance condition $k_{p} \sigma_{z}=1$. The transverse beam size is chosen equal to the transverse matching condition as defined below. For $\alpha \leq 1$ there is no full blow-out and the ion column model, described in the next section, cannot be applied. In this case, as justified in the Appendix, the wakefield linear equations in electrostatic approximation can be used to retrieve the focusing force acting on the beam. The envelope equation reads

$$
\sigma_{x, y}^{\prime \prime}+\frac{\Lambda \mathcal{Z}}{4 \gamma \sigma_{x, y}}=\frac{\epsilon_{n, \mathrm{rms}}^{2}}{\gamma^{2} \sigma_{x, y}^{3}}
$$

leading to the matching condition for a low density driver

$$
\sigma_{(x, y) D}=\sqrt{\frac{4}{\gamma \Lambda \mathcal{Z}}} \epsilon_{n} .
$$

Here $\Lambda=\alpha k_{p}^{2} \sigma_{x, y}^{2}$ is the normalized charge per unit length and $\mathcal{Z}$ is defined as the longitudinal normalized plasma response function

$$
\mathcal{Z}=-\left.\frac{e Z(\xi)}{\alpha m_{e} c^{2}}\right|_{\xi=0} ;
$$

where $Z(\xi)$ is the longitudinal part of the Green's response function of the plasma [12] and $\xi=0$ corresponds to the drive beam center. For a bi-Gaussian beam:

$$
\mathcal{Z}=\sqrt{\pi / 2} k_{p} \sigma_{z} e^{-k_{p}^{2} \sigma_{z}^{2}} \operatorname{erfi}\left(k_{p} \sigma_{z} / \sqrt{2}\right) ;
$$

where erfi is the imaginary error function.

\section{WITNESS MATCHING CONDITIONS}

The linear regime is usually not considered suitable for PWFA for several reasons [21]. Among them, it is noticed how the focusing generated by a driver in linear regime is not suitable for preserving witness quality. In blow-out schemes, where the witness is injected inside the ion column, the witness transverse behavior follows the ion column model $[14,18,19]$ both for PWFA and LWFA. In this model, the focusing fields are derived assuming that the plasma electrons are totally blown-out, generating a channel of unshielded ions. The witness is entirely contained in this ion channel, where the plasma has a purely electrostatic focusing force. The resulting matching condition for a driver with Lorentz factor $\gamma$ and transverse normalized emittance $\epsilon_{n}$ is [19]

$$
\sigma_{x, y}=\sqrt[4]{\frac{2}{\gamma}} \sqrt{\frac{\epsilon_{n}}{k_{p}}} .
$$

In the presented case, the witness is injected in a region where the transverse force generated by the driver is low and the overall stability of the focusing is due to the beam loading effect. This configuration is very similar to the selffocusing experienced by a single beam of high density that is injected in neutral plasma. It is well known in literature that in this case the beam generates a blow-out region that focuses the beam itself [14], but it also experiences a significant head erosion effect, leading to a catastrophic quality degradation for long distances [22,23]. Simulations show that the witness effectively generates a blow-out region in its wake, as represented in Fig. 1. Nevertheless, the simulations suggest also that the beam doesn't experience head erosion and the emittance is fully preserved exploiting the matching condition Eq. (1).

In Fig. 5 are displayed the trajectories of sample plasma particles crossing the witness and those particles are deflected from the axis by a transverse field. A small plasma electron speed is assumed in correspondence of the witness core. This is justified by the fact that in linear and weakly nonlinear regimes, the longitudinal electron speed is generally negligible [24]. Since the electrons are deflected by the witness action, their transverse speed is canceled in the core and is reasonably small in the surrounding region. Therefore, the focusing field is approximated to purely electrostatic, neglecting the focusing force generated by plasma magnetic field. In literature it is pointed out that the passage of an ultrashort beam with an ultrahigh density causes an immediate plasma electron blowout [25]. In this work there is clear information about the local plasma density before the witness $n=n_{p}$. In our case, this is an approximation since the action of driver is neglected. Even so, the witness is localized close to the crest of the accelerating field, where the linear theory provides that the plasma is locally neutral. It was verified 


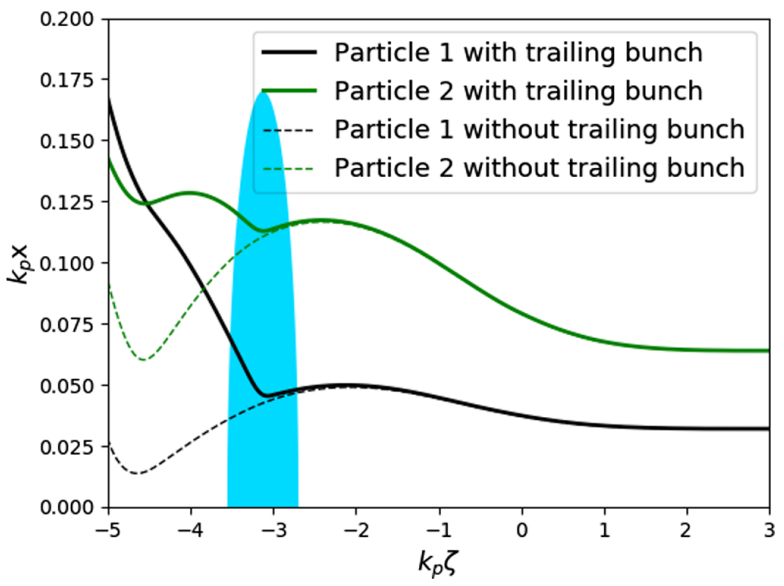

FIG. 5. A detail of the trajectories (continuous lines) of two sample particles crossing the witness (blue). To remark the witness effect also the trajectories of the same particles without witness (dashed lines) are displayed.

that the residual non-neutral plasma before the witness has the only effect to focus the head 1 . The high density witness generates a full blow-out, so after the witness $n=0$. Despite having no clue about the behavior of the plasma density inside the beam itself, but it is reasonable to assume that it is constant and, on average, $n=n_{p} / 2$. The total charge density can be evaluated as the sum of the charge of the background ion density and the local electron plasma density $\rho=e n_{p}-e n_{p} / 2=e n_{p} / 2$. Applying the ion column model to our case it comes out

$$
E_{r}=\frac{n_{p} e}{4 \varepsilon_{0}} r
$$

The focusing strength of this channel is

$$
K=\frac{k_{p}^{2}}{4 \gamma}
$$

Inserting Eq. (9) in the envelope equation Eq. (1) is retrieved. The apparent lack of head erosion in this model is due to a residual focusing force of the driver acting on the witness head.

\section{PARAMETRIC STUDIES}

In order to consolidate the results obtained, several parametric scans were performed to prove the validity of Eq. (9) in a wider range of scenarios. The evolution of the $\beta$-function in all investigated cases is plotted in Fig. 6. In the first scan the normalized emittance of the witness at the injection has been varied, recalibrating also the spot size in order to have the same $\beta$-function at the injection. The beam parameters are listed in Table II.
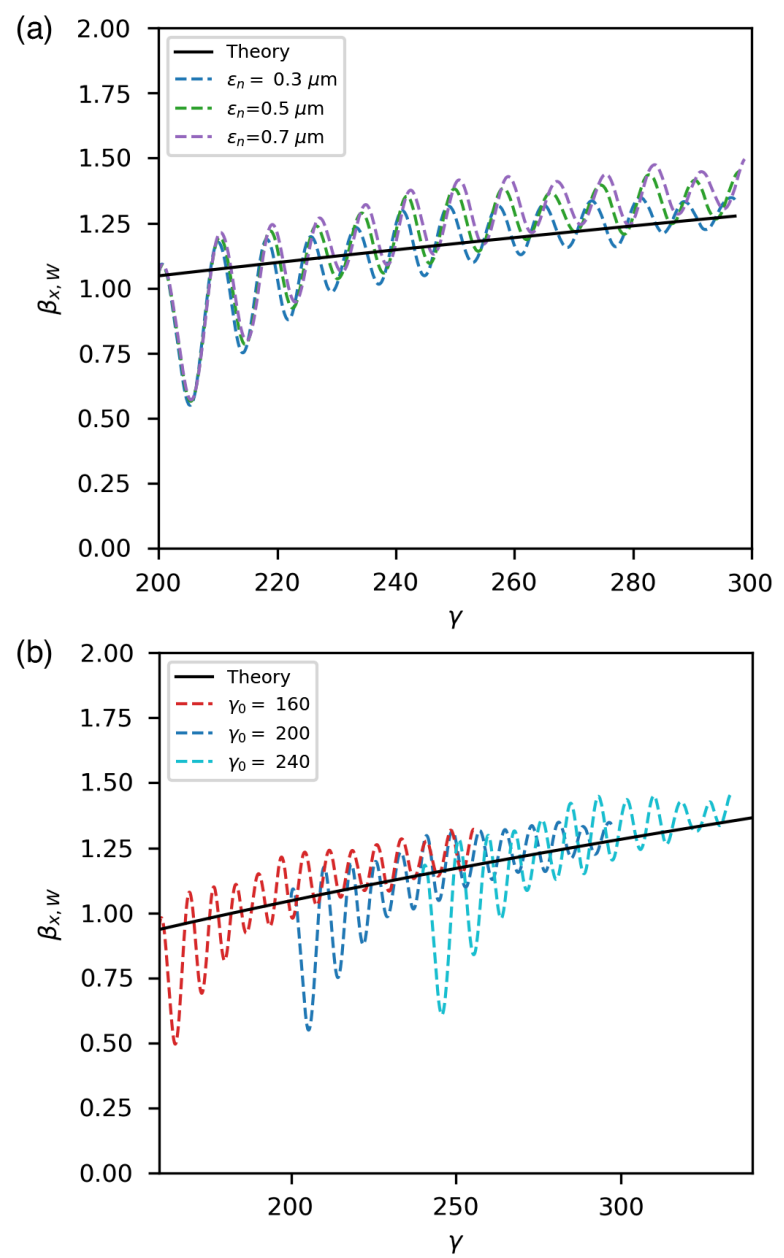

(c)

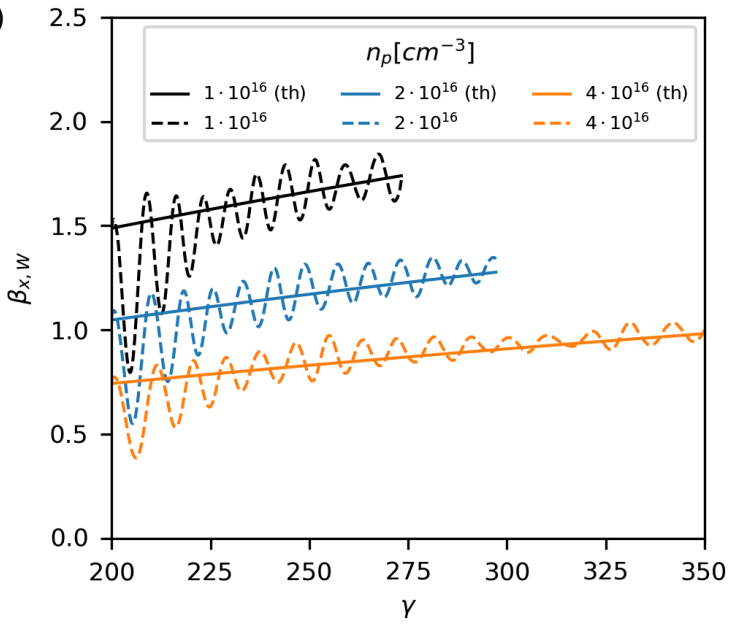

FIG. 6. Evolution of witness $\beta$-function versus bunch energy for different working points. In the first working point scan (a) the witness emittance has been varied preserving the $\beta$-function at the injection. In the second scan (b) the injection energy of the witness has been varied preserving the $\beta$-function at the injection. In the third scan (c) the working point has been redesigned for background plasma densities $n_{p}=1 \times 10^{16} \mathrm{~cm}^{-3}$ (black) and $n_{p}=4 \times 10^{16} \mathrm{~cm}^{-3}$ (orange). 
TABLE II. First parametric scan.

\begin{tabular}{lcc}
\hline \hline & Driver & Witness \\
\hline$Q[\mathrm{pC}]$ & 200 & 10 \\
$\gamma$ & 200 & 200 \\
$\epsilon_{n}[\mu \mathrm{m}]$ & 17 & $0.3-0.5-0.7$ \\
$\sigma_{r}[\mu \mathrm{m}]$ & 10.3 & $1.26-1.63-.92$ \\
$\sigma_{z}[\mu \mathrm{m}]$ & 37.2 & 3 \\
$\sigma_{E}[\%]$ & 0.1 & 0.1 \\
\hline \hline
\end{tabular}

TABLE III. Second parametric scan.

\begin{tabular}{lcc}
\hline \hline & Driver & Witness \\
\hline$Q[\mathrm{pC}]$ & 200 & 10 \\
$\gamma$ & 200 & $160-200-240$ \\
$\epsilon_{n}[\mu \mathrm{m}]$ & 17 & 0.3 \\
$\sigma_{r}[\mu \mathrm{m}]$ & 10.3 & $1.26-1.63-1.92$ \\
$\sigma_{z}[\mu \mathrm{m}]$ & 37.2 & 3 \\
$\sigma_{E}[\%]$ & 0.1 & 0.1 \\
\hline \hline
\end{tabular}

TABLE IV. Working point $n_{p}=1 \times 10^{16} \mathrm{~cm}^{-3}$.

\begin{tabular}{lcc}
\hline \hline & Driver & Witness \\
\hline$Q[\mathrm{pC}]$ & 200 & 10 \\
$\gamma$ & 200 & 200 \\
$\epsilon_{n}[\mu \mathrm{m}]$ & 17 & 0.3 \\
$\sigma_{r}[\mu \mathrm{m}]$ & 12.3 & 1.49 \\
$\sigma_{z}[\mu \mathrm{m}]$ & 52.5 & 3 \\
$\sigma_{E}[\%]$ & 0.1 & 0.1 \\
\hline \hline
\end{tabular}

In the second scan the energy of the witness at the injection was changed, recalibrating also the spot size in order to have the same $\beta$-function at the injection. The beam parameters are listed in Table III.

In the third scan the working points were redesigned assuming a background plasma density $n_{p}=1 \times 10^{16} \mathrm{~cm}^{-3}$ and $n_{p}=4 \times 10^{16} \mathrm{~cm}^{-3}$. Maintaining unaltered the charge and the energy of the driver and the witness, their shapes were redesigned in order to follow the same criteria that were used to design the main working point. The bunch length has been varied in order to keep $k_{p} \sigma_{z}=1$, thus preserving the value of $\mathcal{Z}$ from Eq. (7). Keeping the

TABLE V. Working point $n_{p}=4 \times 10^{16} \mathrm{~cm}^{-3}$.

\begin{tabular}{lcc}
\hline \hline & Driver & Witness \\
\hline$Q[\mathrm{pC}]$ & 200 & 10 \\
$\gamma$ & 200 & 200 \\
$\epsilon_{n}[\mu \mathrm{m}]$ & 17 & 0.3 \\
$\sigma_{r}[\mu \mathrm{m}]$ & 8.7 & 1.06 \\
$\sigma_{z}[\mu \mathrm{m}]$ & 26.3 & 3 \\
$\sigma_{E}[\%]$ & 0.1 & 0.1 \\
\hline \hline
\end{tabular}

emittance constant and evaluating the matching spot size from Eq. (5), the normalized bunch density at the injection $\alpha=1$. As well the witness spot size at the injection was varied in order to follow the matching condition Eq. (1). The beam parameters for these two cases are listed in Table IV and V respectively.

\section{CONCLUSION}

By means of the plasma cold fluid equations, a working point has been designed for beam driven plasma wakefield acceleration that guarantees promising results for preserving the accelerated beam quality. This innovative technique exploits the transverse beam loading effect in order to preserve the beam emittance. In order to achieve this result, the working point was designed assuming a low density driver that generates a linear field. The witness is injected in the crest region of the accelerating field where, according to the plasma cold fluid equations, is expected a very low focusing field generated by the driver. Besides, the accelerated beam was injected with a very high density that guarantees a high transverse beam loading effect. Assuming some approximations on the plasma behavior, that were verified ex post by means of numerical simulations, the working point design was also fully characterized from an analytical point of view. To conclude, several parametric scans have been performed in order to verify the ability to predict the witness transverse behavior in all the investigated scenarios. The relative simplicity in the design, together with the encouraging results, opens new interesting scenarios in the use of this acceleration technique.

\section{ACKNOWLEDGMENTS}

This work was supported by the European Union's Horizon 2020 research and innovation programme under grant agreement No. 653782.

\section{APPENDIX: DRIVER MATCHING CONDITION IN LINEAR APPROXIMATION}

The matching condition is found deriving a stationary solution of the transverse envelope equation [19]

$$
\sigma_{x, y}^{\prime \prime}+K \sigma_{x, y}=\frac{\epsilon_{n, x}^{2}}{\gamma^{2} \sigma_{x, y}^{3}}
$$

for linear plasma wakefields. For sake of simplicity the adiabatic damping is neglected. The space charge forces are also neglected since in the plasma the beam is emittance dominated. Here $\epsilon_{n}$ is the transverse normalized beam emittance and $K$ is the average focusing strength experienced by the beam electrons 


$$
K=-\frac{1}{\sigma_{x, y}^{2}} \frac{\left\langle x F_{x, \mathrm{ext}}\right\rangle}{c \beta_{z} p_{z}} .
$$

The derivation of $K$ in linear regime assumes the electrostatic approximation, namely, the plasma current induced focusing fields are negligible respect to the electrostatic fields $|\rho| \gg\left|J_{z} / c\right|$. This assumption has been proved to be rigorously valid in literature for low charge (or low density) beams [24]. Recalling the Green's function solution for the linear plasma response to an arbitrary relativistic charge beam of the form $\rho_{b}(r, \xi)=\rho_{\perp}(r) \rho_{\|}(\xi)$ where $\rho_{b}(r, \xi)=$ $-e n_{b}(r, \xi)$, the transverse wakefield can be written

$$
W_{\perp}=\left[E_{r}-c B_{\theta}\right](r, \xi)=R^{\prime}(r) Z(\xi) ;
$$

and the perturbed plasma density $n_{1}=n_{e}-n_{p}$ where $n_{e}$ is the electron density and $n_{p}$ is the background density

$$
n_{1}(r, \xi)=\frac{\epsilon_{0} k_{p}^{2}}{e} Z(\xi) n_{b \perp}(r) .
$$

Maxwell equations and Eq. (A4) can be combined in order to obtain a differential equation for $R^{\prime}(r)$

$$
\frac{\partial}{\partial r}\left[r R^{\prime}(r)\right]=-r k_{p}^{2} n_{b \perp}(r)
$$

that for bi-Gaussian beam can be integrated as

$$
R^{\prime}(r)=\frac{k_{p}^{2} \sigma_{r}^{2}}{2 r}\left[1-e^{-r^{2} / \sigma_{r}^{2}}\right] .
$$

Then $K=\alpha \mathcal{Z}(\xi) k_{p}^{2} / 4 \gamma$ where

$$
\mathcal{Z}(\xi)=-\frac{e Z(\xi)}{\alpha m_{e} c^{2}} ;
$$

is the longitudinal normalized plasma response function. From the definition of $\alpha=\Lambda /\left(k_{p}^{2} \sigma_{x, y}^{2}\right)$, the external focusing effect has an implicit dependence on the beam shape. Assuming a fixed $\Lambda$ during all the propagation and negligible beam deformations Eq. (4) can be derived. This equation at $\xi=0$ (beam core) admits the quasistationary solution Eq. (5).

[1] S. Bini et al., Development of x-band accelerating structures for high gradients, Chin. Phys. C 36, 639 (2012).

[2] T. Tajima and J. Dawson, Laser Electron Accelerator, Phys. Rev. Lett. 43, 267 (1979).

[3] P. Chen, J. Dawson, R. Huff, and T. Katsouleas, Acceleration of Electrons by the Interaction of a Bunched Electron Beam with a Plasma, Phys. Rev. Lett. 54, 693 (1985).
[4] H. Kim, K. Pae, H. Cha, I. Kim, T. Yu, J. Sung, S. Lee, T. Jeong, and J. Lee, Enhancement of Electron Energy to the Multi-gev Regime by a Dual-Stage Laser-Wakefield Accelerator Pumped by Petawatt Laser Pulses, Phys. Rev. Lett. 111, 165002 (2013).

[5] M. Litos et al., High-efficiency acceleration of an electron beam in a plasma wakefield accelerator, Nature (London) 515, 92 (2014).

[6] F. Tsung, R. Narang, W. Mori, C. Joshi, R. Fonseca, and L. Silva, Near-Gev-Energy Laser-Wakefield Acceleration of Self-Injected Electrons in a Centimeter-Scale Plasma Channel, Phys. Rev. Lett. 93, 185002 (2004).

[7] M. Ferrario, D. Alesini, A. Bacci, M. Bellaveglia, R. Boni, M. Boscolo, P. Calvani, M. Castellano, E. Chiadroni, A. Cianchi et al., Laser comb with velocity bunching: Preliminary results at sparc, Nucl. Instrum. Methods Phys. Res., Sect. A 637, S43 (2011).

[8] R. Pompili et al., Beam manipulation with velocity bunching for PWFA applications, Nucl. Instrum. Methods Phys. Res., Sect. A 829, 17 (2016).

[9] S. Romeo, Beam loading assisted matching working point for PWFA beam driven experiment at SPARC_LAB, Ph.D. thesis, La Sapienza, University of Rome, 2017.

[10] V. K. B. Olsen, E. Adli, and P. Muggli, Emittance preservation of an electron beam in a loaded quasi-linear plasma wakefield, arXiv:1710.04858.

[11] T. Katsouleas, S. Wilks, P. Chen, J. Dawson, and J. Su, Beam loading in plasma accelerators, Part. Accel. 22, 81 (1987).

[12] W. Lu, C. Huang, M. Zhou, W. Mori, and T. Katsouleas, Limits of linear plasma wakefield theory for electron or positron beams, Phys. Plasmas (1994-present) 12, 063101 (2005).

[13] J. Rosenzweig, B. Breizman, T. Katsouleas, and J. Su, Acceleration and focusing of electrons in two-dimensional nonlinear plasma wake fields, Phys. Rev. A 44, R6189 (1991).

[14] N. Barov and J. Rosenzweig, Propagation of short electron pulses in underdense plasmas, Phys. Rev. E 49, 4407 (1994).

[15] F. Massimo, S. Atzeni, and A. Marocchino, Comparisons of time explicit hybrid kinetic-fluid code architect for plasma wakefield acceleration with a full pic code, J. Comput. Phys. 327, 841 (2016).

[16] R. Courant, K. Friedrichs, and H. Lewy, Über die partiellen differenzengleichungen der mathematischen physik, Math. Ann. 100, 32 (1928).

[17] M. Surkova, W. Tierens, I. Pavlenko, D. Van Eester, G. Van Oost, and D. De Zutter, 3-d discrete dispersion relation, numerical stability, and accuracy of the hybrid fdtd model for cold magnetized toroidal plasma, IEEE Trans. Antennas Propagat. 62, 6307 (2014).

[18] T. Mehrling, J. Grebenyuk, F. Tsung, K. Floettmann, and J. Osterhoff, Transverse emittance growth in staged laserwakefield acceleration, Phys. Rev. Accel. Beams 15, 111303 (2012).

[19] M. Ferrario, Injection, extraction and matching, arXiv: 1705.10564 .

[20] H. Wiedemann, Particle Accelerator Physics (Springer, New York, 2015). 
[21] J. Rosenzweig, P. Colestock, N. Barov, and E. Colby, A linear collider based on nonlinear plasma wake-field acceleration, eConf 960625, 394 (1996).

[22] N. Barov, J. Rosenzweig, M. Conde, W. Gai, and J. Power, Observation of plasma wakefield acceleration in the underdense regime, Phys. Rev. Accel. Beams 3, 011301 (2000).

[23] M. Zhou et al., Beam head erosion in self-ionized plasma wakefield accelerators, in 2007 IEEE Particle Accelerator
Conference (PAC) (IEEE, New York, 2007), pp. 3064 3066.

[24] W. Lu, C. Huang, M. Zhou, M. Tzoufras, F. Tsung, W. Mori, and T. Katsouleas, A nonlinear theory for multidimensional relativistic plasma wave wakefields, Phys. Plasmas (1994-present) 13, 056709 (2006).

[25] G. Stupakov, B. Breizman, V. Khudik, and G. Shvets, Wake excited in plasma by an ultrarelativistic pointlike bunch, Phys. Rev. Accel. Beams 19, 101302 (2016). 